\title{
Role of Multi Detector Computed Tomography in the Evaluation of Glenoid Track in Patients with Anterior Instability and Its Correlation with Arthroscopy
}

\author{
Prabhu Radhan ${ }^{1}$, Yoshitha Siripurapu², Prakash Ayyadurai ${ }^{3}$, Bhaskar Raj ${ }^{4}$, Arumugam Sivaraman $^{5}$, \\ Rajoo Ramachandran ${ }^{6}$
}

\begin{abstract}
${ }^{1}$ Department of Radiodiagnosis, Sri Ramachandra Institute of Higher Education and Research, Chennai, Tamilnadu, India. ${ }^{2}$ Department of Radiodiagnosis, Sri Ramachandra Institute of Higher Education and Research, Chennai, Tamilnadu, India. ${ }^{3}$ Department of Orthopaedics, Sri Ramachandra Institute of Higher Education and Research, Chennai, Tamilnadu, India. ${ }^{4}$ Department of Radiodiagnosis, Sri Ramachandra Institute of Higher Education and Research, Chennai, Tamilnadu, India. ${ }^{5}$ Department of Orthopaedics, Sri Ramachandra Institute of Higher Education and Research, Chennai, Tamilnadu, India. ${ }^{6}$ Department of Radiodiagnosis, Sri Ramachandra Institute of Higher Education and Research, Chennai, Tamilnadu, India.
\end{abstract}

\section{ABSTRACT}

\section{BACKGROUND}

Glenoid track is a new classification system developed to biomechanically quantify the effects of a combined glenoid and humeral head bony defects on instability that can be utilized to assess the risk of engagement of a Hill-Sachs lesion in a patient with anterior shoulder instability. The aim of the study is to evaluate the humeral head engagement on the glenoid by utilizing glenoid track method and to compare the results with the arthroscopic findings.

\section{METHODS}

We performed a prospective study of 31 patients with recurrent shoulder dislocation over a period of two years. A 64 slice CT scanner was used. Using post processing technique, the glenoid track was calculated to classify the shoulders as on-track or off-track.

\section{RESULTS}

The glenoid track and concurrent Hill Sachs interval were calculated into 4 entities"Off-track" lesion with <25\% glenoid bone loss [41. 94\%], > 25\% bone loss [6. 45\%], "On-track" lesion with $<25 \%$ glenoid bone loss [51. 61\%] and $>25 \%$ glenoid bone loss [0].

\section{CONCLUSIONS}

The glenoid track concept postulated that a Hill-Sachs lesion outside this track was at a high risk for engagement and thus recurrent instability. CT measurement as a predictor of off-track and on-track lesion, using the differences between Hill-Sachs and glenoid track had a p value of 0.0001 . The glenoid track concept is important to assess the overall risk for engagement prior to surgery and help in guiding surgical decision making such as bony augmentation procedure.

\section{KEY WORDS}

Glenoid Track, Off Track Lesion, On Track Lesion, Glenoid Bone Loss, Arthroscopic Bankart Repair
Corresponding Author: Yoshitha Siripurapu, \#54-20-9-7A, Thimmarasu Street, Srinagar Colony, Ring Road, Vijayawada-520008, Andhra Pradesh, India. E-mail: yoshitha.siripurapu16@gmail.com

DOI: 10. 14260/jemds/2019/864

Financial or Other Competing Interests: None.

How to Cite This Article:

Radhan P, Siripurapu Y, Ayyadurai $P$, et al. Role of multi detector computed tomography in evaluation of the glenoid track in patients with anterior instability and its correlation with arthroscopy. J. Evolution Med. Dent. Sci. 2019;8(52): 3997-4001, DOI:10. 14260/jemds/2019/864

Submission 14-11-2019,

Peer Review 13-12-2019,

Acceptance 17-12-2019,

Published 30-12-2019. 


\section{BACKGROUND}

Dislocation of the shoulder occurs following traumatic injury, such as a fall, road traffic accident or as a result of overuse/repetitive strain from sports. The shoulder is in its weakest position when it is abducted and externally rotated. Sporting injuries and motor vehicle collisions being the most common causes. It is a ball and socket type of articulation between the glenoid fossa of the scapula and the head of the humerus. [1] In a shoulder dislocation, there is separation of the humerus from the glenoid of the scapula at the glenohumeral joint. There is increased incidence in patients who have had a previous shoulder injury, and particularly in those who have dislocated previously. The process of dislocation is massively disruptive to the labrum, joint capsule, supporting ligaments, and muscles. This is particularly true of anterior dislocations where there can be an injury to the anterior capsule, anterior labrum, or biceps tendon, or a combination. Shoulder dislocations are usually divided according to the direction in which the humerus exits the joint, which includes Anterior dislocation, accounting for $>95 \%$ [3 subtypes include- subcoracoid (majority) , subglenoid (1/3) , subclavicular], Posterior dislocation 2-4\% and Inferior dislocation (luxatio erecta) $<1 \%$. Anterior shoulder dislocation is by far the commonest type of dislocation and usually results from forced abduction, external rotation and extension. [1] Predisposing factors for recurrent anterior shoulder dislocation includes flattened or shallow anterior/ anteroinferior glenoid bony contour. ${ }^{[2]}$ The essential lesion of an anterior shoulder dislocation results in a humeral bone loss (Hill Sachs lesion) or a midsubstance/capsular tear or a Bankart lesion and its variants.

The Hill-Sachs lesion is classically described as a compression fracture of the postero-superolateral humeral head in association with anterior instability or dislocation of the glenohumeral joint. Joseph-François Malgaigne first documented this lesion in his 1855 thesis, and Eve, [3] expounded on it with the first case report and its association with a glenohumeral dislocation. It was radiographically described by Hill and Sachs as a line of condensation on the internal rotation shoulder radiograph and was attributed to the impression of the dense cortical glenoid on the humeral head during an anterior dislocation event. [3] The incidence of Hill-Sachs lesions in anterior shoulder instability ranges from $38 \%$ to $88 \%$ and is associated with up to $100 \%$ of recurrent dislocations. [3] Management of the Hill Sachs and the Bankart lesions [So called bi-polar bone loss] is essential for stability of the joint and for prevention of further dislocations. Surgery for a Bankart lesion is highly recommended for active athletes, even after a single dislocation, especially those involved in contact sports, which increase the likelihood of a second shoulder dislocation and shoulder joint injury. Repair of the labrum followed by the reattachment of the glenohumeral ligament to the glenoid rim mostly via arthroscopic surgery is done. The success rate of surgical repair is approximately $90 \%$ and patients are able to return to their regular activities.

With a Hill-Sachs lesion that occupies $>30 \%$ of the humeral head surface, as established by CT or MRI scan, surgery is required due to the high instability of the shoulder. Surgical techniques vary: Allograft (transplant of a small bone tissue) into the lesion of the humeral head; remplissage
(French translation for "fill-in") in which a burr is introduced to decorticate the Hill-Sachs lesion and a large rotator cuff anchor is inserted into the bony defect; humeral rotation osteotomy or removal of bone parts; hemi-arthroplasty and total shoulder arthroplasty are only used in patients over 50 years of age. The evaluation of the bipolar bone loss [Glenoid and Hill Sachs], by MDCT is essential for categorizing the types of glenoid bone loss in relation with the Hill Sachs thereby enabling the treatment planning for stability of the joint. The glenoid track concept has been helpful for the assessment of the bi-polar bone loss for pre-operative planning. Multidetector Computed Tomography [MDCT] has been an integral modality to evaluate the glenoid track for biomechanically quantifying the effects of a combined glenoid and humeral head bony defects on instability.

\section{METHODS}

A prospective observational study was conducted in department of radiology in Sri Ramachandra Medical College during the time period August 2015 to August 2017 in 31 patients who were referred for CT SHOULDER who were diagnosed with recurrent anterior dislocations. 31 patients in whom bipolar bone loss was detected on CT imaging were included in this study. Institutional Ethics committee approval was obtained for the study proposal and informed consent was obtained in all patients.

\section{Inclusion Criteria}

Patients with anterior shoulder instability diagnosed with $\mathrm{x}$ ray and MRI, who have a clinical history of self-reduction for recurrent shoulder dislocation and patients who would benefit with surgical treatment

\section{Exclusion Criteria}

Patients with first time dislocation, previous shoulder surgery and who have contraindications to CT imaging.

\section{CT Protocol}

All patients who were referred for CT shoulder are first explained about the procedure, detailed clinical history was taken, previous medical records checked and then written informed consent was obtained. Preoperative multidetector computed tomography imaging was used to determine the glenoid bone loss, Hill-Sachs lesion [Size and location] and to measure the glenoid track to classify the shoulders as ontrack or off-track. Outcomes were correlated with the arthroscopic findings.

\section{Procedure}

Computed Tomography imaging of bilateral shoulder was performed in all patients.

\section{Procedure for Calculating the Glenoid Track}

The patient is placed in the CT gantry and both shoulders are always placed in the scanning field. With a single scan, the data of both shoulders are recorded so that we can utilize the data of both shoulders. We used the contralateral shoulder as a reference. After taking AP and Lateral scout and serial 
helical sections of the shoulder joint with each slice thickness of $5 \mathrm{~mm}$ is taken. Recon- $0.625 \mathrm{~mm}$, using KV-120, mAs- 400 . Using bone logarithm setting, post processing technique by advanced workstation ADW-4. 4 version the glenoid track was calculated. The Hill Sachs lesion was measured on axials and glenoid bone loss on thick MIP.

\section{Measuring the Glenoid Bone Loss}

First, we create an end face view of the glenoid, using thick MIP [3 mm]. We used the best fit circle method to evaluate the width of the glenoid on normal side. Using the normal side as reference, the glenoid defect width on abnormal side is calculated. Glenoid defect/bone loss = glenoid width on normal side- glenoid width on abnormal side Glenoid track on normal side is calculated as $83 \%$ of the normal glenoid width. The defect width is then deducted from the glenoid track of normal side to arrive at the glenoid track of the abnormal side. [2, 4]

\section{Measuring the Hill Sachs Lesion}

Using the best fit circle similar to the glenoid width measurement, the Hill Sachs interval is calculated by adding the Hill Sachs lesion with the bony bridge. It is important to note that there is typically an intact bone bridge between the rotator cuff attachments and the medial margin of the HillSachs lesion. This bone bridge (BB) width plus the width of the Hill-Sachs lesion equals what we call the Hill-Sachs Interval (HSI), whose medial margin is the critical point in determining if a Hill-Sachs lesion is non-engaging or engaging. If the medial margin of a Hill-Sachs lesion is within the glenoid track, there is bone support adjacent to the HillSachs, and the Hill-Sachs lesion is "non-engaging" or "on track". If the medial margin of the Hill-Sachs lesion is more medial than the glenoid track, there is no bone support, and the Hill-Sachs lesion is "engaging" or "off track". On the basis of our quantitative method, we have correlated with the intra-operative findings for all patients with anterior instability, with bipolar bone loss.

\section{Statistical Method}

Data analysis were done using IBM SPSS version 16. Descriptive statistics like frequency and percentage were used. Inferential statistical tests like Intra class correlation was done. The pre-op measurements on CT were analysed intra operatively [arthroscopically] and hence were found to be the same. $p$ value $<0.05$ was regarded as indicative of a statistically significant difference.

\section{RESULTS}

In our study 31 patients who had recurrent anterior shoulder dislocation were included, out of which 30 are males and one female. Majority of patients presented in the 2nd decade followed by 3rd and 4th decades of life. The age at presentation varied from 18 to 53 yrs. Multidetector Computed Tomography imaging of bilateral shoulder was performed in all patients. Using the post processing technique, the glenoid track was calculated.
The mean glenoid diameter was $28 \mathrm{~mm}$ and standard deviation was 2. 33 [Range 24. 3-33 mm], the mean glenoid bone loss percentage was $16 \%$ [Range 0\%-27. 07\%]. The mean Hill Sachs index was $18 \mathrm{~mm}$ [Range 8-22. $8 \mathrm{~mm}$ ]. The mean glenoid track was $19 \mathrm{~mm}$ [Range 14. 8-24.1 mm]. Interand intrareader reliability in the CT imaging assessment was determined with the intra class correlation coefficient. Intrareader Correlation was moderate to strong in assessment of Glenoid bone loss $(r=0.86)$ \& Hill-Sachs interval $(r=0.75)$. A senior sport medicine surgeon with two assistant surgeons analysed the radiographic parameters and calibrated the measurements intra-operatively on all patients independently. On the basis of our quantitative method, we have correlated with the intra-operative findings for all patients with anterior instability, with bipolar bone loss. In our cohort, all the categories of the on track and off-track population was similar when calibrated intra-operatively and thus the treatment was planned.

In our study, 16 patients [51. 61\%] with on track lesions with $<25 \%$ of bone loss were treated by Bankart repair [table 2]. 13 patients [41. 94\%] with off track lesions with $<25 \%$ of bone loss were treated with Bankart repair and remplissage procedure. 2 patients [6. 45\%] with off track lesions with $>25 \%$ of bone loss were treated with Latarjet procedure. We did not have patients with on track lesion with $>25 \%$ bone loss in our cohort. On the basis of our quantitative method, we have correlated with the intra-operative findings for all patients with anterior instability, with bipolar bone loss. We categorized all our anterior instability patients, regardless of the degree of bipolar bone loss, into one of 4 categories (Table 1) and the treatment paradigm was planned (Table 2)

\begin{tabular}{|c|c|c|}
\hline Group & Glenoid Defect & Hill-Sachs \\
\hline 1 & $<25 \%$ & Non-engaging/on track \\
\hline 2 & $<25 \%$ & Engaging/off track \\
\hline 3 & $\geq 25 \%$ & Non-engaging/on track \\
\hline 4 & $\geq 25 \%$ & Engaging/off track \\
\hline \multicolumn{3}{|c|}{ Table 1. Anterior Instability Categories } \\
\hline
\end{tabular}

\begin{tabular}{|c|c|}
\hline Group & Treatment \\
\hline 1 & Arthroscopic Bankart repair \\
\hline 2 & Arthroscopic Bankart repair plus remplissage \\
\hline 3 & Latarjet \\
\hline 4 & $\begin{array}{c}\text { Latarjet } \pm \text { humeral-sided procedure (Humeral bone graft or } \\
\text { remplissage), depending upon engagement of Hill Sachs. }\end{array}$ \\
\hline \multicolumn{2}{|c|}{ Table 2. Surgical Treatment Paradigm } \\
\hline
\end{tabular}

\section{DISCUSSION}

The incidence of Hill-Sachs lesions in anterior shoulder instability ranges from $38 \%$ to $88 \%$ and is associated with up to $100 \%$ of recurrent dislocations. Management of the Hill Sachs and the Bankart lesions [so called bi-polar bone loss] is essential for stability of the joint and for prevention of further dislocations. The glenoid track concept has been helpful for the assessment of the bi-polar bone loss for pre-operative planning.

In recent years, efforts have been made to identify the morphology, extent, location, and interaction of the bone losses for better surgical outcome. The role of preoperative imaging study is not only quantifying glenoid bone loss but to assess the glenoid shape and morphology to facilitate 
surgeons in stabilizing the shoulder. [3] In this view, 3DCT is the most recommended preoperative imaging study for bony tissue which provides critical and substantial information of the glenoid. Several authors described glenoid bone loss measurement using preoperative imaging, especially using unilateral 2D and 3DCT. [5, 6, 7, 8, 9, 10, 11, 12] It is generally accepted that CT, especially en face view of 3DCT images, is the most preferable preoperative imaging study for quantification of glenoid bone loss. However, several authors are making an attempt to replace CT with MRI in order to reduce cost for imaging studies and avoid possible radiation exposure. $[13,14,15,16]$ Owens and co-workers proposed a unique method of obviating use of CT using MRI. [15]

The role of preoperative imaging study is, first of all, to assess the glenoid shape and morphology in shoulders with recurrent anterior instability in addition to quantifying glenoid bone loss [5]. In this view, 3DCT is the most recommended preoperative imaging study for bony tissue which provides critical and substantial information of the glenoid. $[5,17,19]$ There are various methods to assess the size of the glenoid bony defect: defect length, width-to-length ratio, glenoid index (i.e., defect width/circle diameter), and Pico method (i.e., defect area/circle area) . We prefer to use the contralateral shoulder as a reference because the difference between the right and left sides is extremely small. According to Jeskeet al., the average area difference between the left and right sides was only $1.8 \%$. We can reliably use the contralateral glenoid as a reference. [4]

Yamamoto et al developed a novel approach and developed the concept of "glenoid track." They were also able to verify their model in 3 patients with anterior instability using 3-dimensional reconstruction computed tomography (CT) images. Itoi and associates [2] examined the exact anatomic relationship between the humeral head and the glenoid in various critical positions, when all the anterior soft-tissue structures were preserved. This investigation was a prelude to the critically important concept of the "glenoid track. "Itoi and associates stated that an intact glenoid track, without significant bone loss, guarantees bone stability. Burkhart and DeBeer's concept of engaging vs non-engaging is completely consistent with Yamamoto and Itoi's concept of the glenoid track. They are complementary concepts in that they both evaluate the interaction of bipolar bone loss during dynamic shoulder function. The presence of an engaging HillSachs lesion can be detected at arthroscopy with the arm in abduction-external rotation, while the glenoid track can be evaluated by either arthroscopy or CT scan.

\section{Relationship of Glenoid Track to Engaging and Non- Engaging Hill-Sachs Lesion}

Itoi and Boileau have stated that all bipolar bone lesions are engaging, due to the fact that engagement was required for formation of the Hill-Sachs lesion. This concept was reinforced by Kurokawa et al, who recommended that the glenoid track rather than dynamic intraoperative assessment be used to assess engaging Hill-Sachs lesions. Dynamic intraoperative assessment is almost always performed before repairing the Bankart lesion. It should be emphasized that the prevalence of a true engaging Hill-Sachs lesion using dynamic arthroscopic assessment after Bankart repair is the same as the one assessed with use of the glenoid track concept. Di Giacomo et al. combined the ideas of an engaging Hill-Sachs lesion and the glenoid track to develop a method of calculating the likelihood that a Hill-Sachs lesion will engage in the functional range of motion, accounting for both the size of the Hill-Sachs lesion and the amount of glenoid bone loss. [20]

\section{Correlation with Arthroscopy}

A study similar to ours, where clinical determination of "engagement" was made for each patient by reviewing the reported intraoperative examination findings and evaluating intraoperative video arthroscopy was done. However they have verified the engagement of the Hill-Sachs lesion under initial diagnostic video arthroscopy before any fluid was introduced into the joint and the number of engaging shoulders in the outside (OUT-E, OUT-Engaging) and inside (IN-NE, IN-Non Engaging) glenoid track groups were recorded. In our cohort, all the categories of the on track and off-track population was similar when calibrated intraoperatively and thus the treatment was planned.

\section{Treatment}

If a lesion is noted to be outside the glenoid track and therefore at increased risk for instability, there are a number of potential surgical treatment options that utilize the principles of this concept. One can augment anterior glenoid bone loss with a Latarjet procedure or an iliac crest bone graft to, in effect, increase the glenoid track size and prevent engagement. Another treatment strategy is to shift the glenoid track so that it contains the entire Hill-Sachs lesion and thus prevents engagement. This can be accomplished using a rotational humeral osteotomy or by tightening the anterior soft tissue structures to limit external rotation and shift the glenoid track medially and superiorly. Video arthroscopy was reviewed to determine engagement through the functional range of motion and the pre-operative values were compared. We have found that the pre-operative CT findings were correlated intra-operatively. We prefer to address significant bipolar bone loss with a Latarjet procedure, in which the coracoid bone graft provides additional stability from the sling effect of the conjoined tendon. With this combination of glenoid track enlargement plus the sling effect, the Latarjet procedure is able to effectively address even large degrees of bipolar bone loss without having to resort to additional humeral-sided procedures (humeral bone graft or remplissage) .

Furthermore, to our knowledge, ours is the first study to evaluate the glenoid track concept in a clinical population using postoperative functional outcomes. In conclusion, the glenoid track concept was recently proposed to evaluate the combined importance of both glenoid and humeral bone loss in shoulder instability. The application of this concept to our clinical cohort demonstrated that the glenoid track was a more accurate predictor of instability compared with isolated glenoid bone loss after primary arthroscopic Bankart repairs. This validation of the glenoid track concept encourages its use in the diagnostic work-up of patients who are undergoing consideration of stabilization for anterior shoulder instability. There were lot of recurrence/ failure rates post operatively when the surgeons were addressing the unipolar bone loss alone. The evaluation of the bipolar bone loss [Glenoid and Hill Sachs ] is essential for appropriate treatment planning. The glenoid track concept introduced by 
Itoi and Yamomato in 2007, has been a novel approach for the assessment of the bi-polar bone loss. The glenoid track concept is important to assess the glenohumeral engagement prior to surgery for appropriate surgical decision making. Multidetector Computed Tomography [MDCT] is the modality to evaluate the glenoid track and to categorise the patients into on-track/engaging and off-track/non-engaging.

\section{CONCLUSIONS}

Glenohumeral engagement was well predicted using the glenoid track method while correlating it intra-operatively. It should be considered as a routine part of preoperative evaluation of all patients taken for arthroscopic anterior stabilization. We conclude that for detection and quantification of the bipolar bone loss, MDCT is accurate and effective. The technique of estimation of glenoid bone loss in our study is easily reproducible and has been proved to have very less intra-observer variations.

\section{REFERENCES}

[1] Manaster BJ, Disler DG, May DA. Musculoskeletal imaging, the requisites. Mosby Inc., 2002.

[2] Metzger PD, Barlow B, Provencher MT, et al. Clinical application of the "Glenoid Track" concept for defining humeral head engagement in anterior shoulder instability. Orthop J Sports Med 2013;1 :2325967113496213.

[3] Sugaya H. Techniques to evaluate glenoid bone loss. Curr Rev Musculoskelet Med 2014;7 (1) :1-5.

[4] Di Giacomo G, Itoi E, Burkhart SS. Evolving concept of bipolar bone loss and the Hill-Sachs lesion: from "engaging/non-engaging" lesion to "on-track/off-track" lesion. Arthroscopy 2014;30 (1) :90-8.

[5] Sugaya H, Moriishi J, Dohi M, et al. Glenoid rim morphology in recurrent anterior glenohumeral instability. J Bone Joint Surg Am 2003;85 (5) :878-84.

[6] Sugaya H, Moriishi J, Kanisawa I, et al. Arthroscopic osseous Bankart repair for chronic recurrent traumatic anterior glenohumeral instability. J Bone Joint Surg Am 2005;87 (8) :1752-60.

[7] Griffith JF, Antonio GE, Tong CW, et al. Anterior shoulder dislocation: quantification of glenoid bone loss with CT. Am J Roentgenol 2003;180 (5) :1423-30.

[8] Griffith JF, Antonio GE, Yung PS, et al. Prevalence, pattern and spectrum of glenoid bone loss in anterior shoulder dislocation: CT analysis of 218 patient. Am J Roentgenol 2008;190 (5) :1247-54.
[9] Griffith JF, Yung PS, Antonio GE, et al. CT compared with arthroscopy in quantifying glenoid bone loss. Am J Roentgenol 2007;189 (6) :1490-3.

[10] Chuang TY, Adams CR, Burkhart SS. Use of preoperative three-dimensional computed tomography to quantify glenoid bone loss in shoulder instability. Arthroscopy 2008;24 (4) :376-82.

[11] Nofsinger C, Browning B, Burkhart SS, et al. Objective preoperative measurement of anterior glenoid bone loss: a pilot study of a computer-based method using unilateral 3-dimensional computed tomography. Arthroscopy 2011;27 (3) :322-9.

[12] Dumont GD, Russell RD, Browne MG, et al. Area-based determination of bone loss using the glenoid arc angle. Arthroscopy 2012;28 (7) :1030-5.

[13] De Filippo M, Castagna A, Steinbach LS, et al. Reproducible noninvasive method for evaluation of glenoid bone loss by multiplanar reconstruction curved computed tomographic imaging using a cadaveric model. Arthroscopy 2013;29 (3) :471-7.

[14] Huijsmans PE, Haen PS, Kidd M, et al. Quantification of a glenoid defect with three-dimensional computed tomography and magnetic resonance imaging: a cadaveric study. J Shoulder Elbow Surg 2007;16 (6) :803-9.

[15] Lee RK, Griffith JF, Tong MM, et al. Glenoid bone loss: assessment with MR imaging. Radiology 2013;267 (2) :496-502.

[16] Stecco A, Guenzi E, Cascone T, et al. MRI can assess glenoid bone loss after shoulder luxation: inter- and intra-individual comparison with CT. Radiol Med 2013;118 (8) :1335-43.

[17] Owens BD, Burns TC, Campbell SE, et al. Simple method of glenoid bone loss calculation using ipsilateral magnetic resonance imaging. Am J Sports Med 2013;41 (3) :622-4.

[18] Bois AJ, Fening SD, Polster J, et al. Quantifying glenoid bone loss in anterior shoulder instability: reliability and accuracy of 2-dimensional and 3-dimensional computed tomography measurement techniques. Am J Sports Med 2012;40 (11) :2569-77.

[19] Rerko MA, Pan X, Donaldson C, et al. Comparison of various imaging techniques to quantify glenoid bone loss in shoulder instability. J Shoulder Elbow Surg 2013;22 (4) :528-34.

[20] Di Giacomo G, De Vita A, Costantini A, et al. Management of humeral head deficiencies and glenoid track. Curr Rev Musculoskelet Med 2014;7 (1) :6-11. 